\title{
3D Differentiation Of Mammosphere Derived Macaca fascicularis's Mammary Stem Cells
}

\author{
Silmi Mariya ${ }^{1 *}$, Fitriya N. Dewi ${ }^{1}$, Irma H Suparto ${ }^{1,4}$, Gregory K. Wilkerson ${ }^{2}$, J. Mark \\ Cline $^{3}$, Permanawati ${ }^{1}$, Diah Iskandriati ${ }^{1}$, I Nengah Budiarsa ${ }^{1}$, Dondin Sajuthi ${ }^{1,5}$ \\ ${ }^{1}$ Primate Research Center, Bogor Agricultural University, Jalan Lodaya II/ 5 Bogor 16151, \\ Indonesia \\ ${ }^{2}$ Michale E. Keeling Center for Comparative Medicine and Research, \\ MD Anderson Cancer Center, Bastrop, Texas, USA \\ ${ }^{3}$ Wake Forest School of Medicine, Medical Center Blvd, Winston-Salem, \\ North Carolina, 27157 United States \\ ${ }^{4}$ Faculty of Mathematic and Nature Science, Bogor Agricultural University \\ ${ }^{5}$ Faculty of Veterinary Medicine, Bogor Agricultural University \\ *Corresponding author : mariyasilmi@gmail.com
}

\begin{abstract}
The mammary gland contains adult stem cells that are capable of self-renewal. This population plays an important role in the development of mammary gland and breast cancer pathogenesis. The studies of mammary stem cells are limited due to the difficulty to acquire and expand adult stem cell population in an undifferentiated state. In this study, we developed mammosphere cultures of nulliparous cynomolgus monkeys (Macaca fascicularis; Mf) as a culture system to enrich mammary stem cells. This species has similarity of mammary gland structure as humans including anatomy, developmental stages, and lobule profile of mammary gland. The use of stem cells from primate animals is essential to bridge the knowledge gaps resulting from stem cell research using rodents for clinical trials in human. Small samples of mammary tissues were collected by surgical biopsy; cells were cultured as monolayer and cryopreserved. Cryopreserved cells were cultured into mammospheres, and the expression of markers for mammary stem cells was evaluated using qPCR. Cells were further differentiated with 3D approaches to evaluate morphology and organoid budding. The study showed that mammosphere culture resulted in an increase in the expression of mammary stem cell markers with each passage. The 3D differentiation in matrigel allowed for organoid formation. Mammary gland stem cells have been successfully differentiated which characterized by CSN2 marker expression and differentiation regulators marker STAT5 and GATA3. The results indicate that mammospheres can be successfully developed derived from breast tissue of nulliparous Mf collected via surgical biopsy. As the mammosphere allows for enrichment of mammary stem cell population, the findings also suggest that a 3-dimensional system is efficient as in-vitro model to study mammary stem cells and a useful system to study mammary differentiation in regards to cancer prevention.
\end{abstract}

Keywords: mammospheres, Macaca fascicularis, mammary stem cells, stem cells 


\section{INTRODUCTION}

Multipotent stem cells, known as adult stem cells, are essential to the maintenance of most tissues in the body throughout life. These cells have the ability to undergo self-renewal to produce two stem cells or can divide in a fashion such that one cell remains a stem cell while the other daughter cell undergoes further differentiation. Adult stem cells are normally only present in small numbers within most tissues following gestational development. Mammary gland development is unique, however, as full differentiation of this organ is only attained at adulthood through pregnancy and lactation (Liu et at., 2005). Consistent with this fact, nulliparous breasts are known to contain large numbers of undifferentiated stem cells (LaMarca and Rosen, 2008; Stingl et al., 2006). Adult mammary glands consist of the lobular and ductal structures composed of three cell lineages: alveolar epithelial cells that line the alveoli and synthesize milk proteins; ductal epithelial cells that line the lumen of the ducts; and myoepithelial cells that form the basal layer of both the ducts and alveoli. Alveolar, ductal, and myoepithelial components of the mammary gland initially originate from a common multipotent adult stem cell, the mammary stem cell (MaSC) (Shackleton et al., 2006).

The mammary gland contains adult stem cells that are capable of self-renewal. This

\section{METHODS}

\section{Breast Tissue Collection}

We conducted breast biopsy on adult nulliparous Macaca fascicularis ( $n=3$; age 5-6 population plays an important role in the development of mammary gland and breast cancer pathogenesis, which is thought to be potential as a target for a transformation caused breast cancer. Study the mechanisms of prevention of breast cancer, has limitation in the existing culture model because it represents only for the population of menopausal women. Long-tailed monkey (Macaca fascicularis) is one of primate species that is widely used in biomedical research. This species has similarity of mammary gland structure as humans including anatomy, endocrinology, developmental stages, histology and lobule profile of mammary gland. The use of stem cells from primate animals is essential to bridge the knowledge gaps resulting from stem cell research using rodents for clinical trials in human. The purpose of this study was to develop an in-vitro system of three-dimensional mammary cells (mammosphere) that can enrich stem cell populations, differentiation of mammary gland stem cells became specific cells in mammary gland. The purpose of this study was to develop an in-vitro system of three-dimensional mammary cells (mammosphere) that can enrich stem cell populations and differentiation of mammary gland stem cells became specific cells in mammary gland.

years) to collect mammary gland tissues. All procedures involving animals were performed at Research Animal Facility-Lodaya, Primate 
Research Center at Bogor Agricultural University (PSSP-IPB), an AAALAC International-accredited facility, following ethics approval from PSSP-IPB Institutional Animal Care and Use Committee. Validation of menstrual cycle of monkeys at the time of biopsy were done by vaginal cytology, following daily observation to identify menstrual bleeding pattern. Breast biopsy were performed on deeply-anesthetized animals; under aseptic condition, subcutaneous tissue (approximately $2 \mathrm{~cm} \times 0.5 \mathrm{~cm}$ in size) that contains mammary glands were collected. Upon removal, the tissues were placed in transport media (DMEM, antibiotics, antifungal). Intensive peri- and post-operative care were performed whereby animals were given analgesics and antibiotics, and closely observed throughout the week following biopsy.

\section{Mammary Gland Cell Culture.}

Mammary tissues obtained from biopsy comprised of adipose and glandular tissues. The texture was relatively hard and therefore difficult to dissociate. Digestion with the enzymes collagenase and hyaluronidase allowed for easier mincing and disaggregation. Cells dissociation were done mechanically and enzymatically according to method previously described (Dey et al., 2009), with slight modifications. The collected tissues were digested in $0.075 \%$ collagenase (Sigma Aldrich, USA) and $1 \mathrm{mg}$ hyaluronidase (Sigma Aldrich, USA), and incubated in a humidified atmosphere at $37^{\circ} \mathrm{C}, 5 \% \mathrm{CO}_{2}$ for $16-18$ hours. The tissues were minced and centrifugated at $500 \mathrm{~g}$ for 10 minutes. Supernatant was removed and resuspended in $10 \mathrm{~mL}$ PBS twice. Cells were resuspended to ensure single-cells suspension was formed. Haemocytometer was used to confirm presence of single cells suspension; viable cells were calculated using trypan blue. Cell suspension was plated at appropriate density in selective medium for mammary epithelial cells (Lonza, USA) and incubated in a humidified atmosphere at $37^{\circ} \mathrm{C}$, $5 \% \mathrm{CO}$. Subculture was performed when cell population reached $80 \%$ confluency.

\section{Mammosphere Culture}

All in vitro experiments were performed in duplicates. Frozen MfMC were thawed; cells were seeded 10.000 cells $/ \mathrm{ml}$ in ultra-low attachment plates. Mammospheres that formed from the original cell plating (primary sphere) were collected and examined for subsequent formation of mammospheres following replating (i.e. secondary sphere and tertiary sphere). Mammospheres were collected weekly by centrifugation. For serial passaging, total number of mammospheres obtained at each passage was counted microscopically, enzymatically dissociated into large single cells, and seeded further in low attachment plates after counting for live cells. Mammosphere Forming Efficiency (MFE) was calculated using the following equation: MFE $(\%)=($ number of mammospheres per well) $/$ (number of cells seeded per well) $\mathrm{x} 100$ (Lombardo et al., 2015).

\section{Differentiation}

Cell differentiation was evaluated using twodimensional (2D) and three-dimensional (3D) approaches. The 2D differentiation was induced by culturing the mammosphere in a 6-well 
tissue culture plate with mammary epithelial cell growth medium (Lonza, USA). Cells were incubated for 7 days, trypsinized and prepared for mRNA extraction. The 3D differentiation was induced by culturing mammospheres in matrigel. Following 7 days incubation, organoids formed were collected and prepared for mRNA extraction.

Quantitative real time reverse transcriptase polymerase chain reaction ( $q R T-P C R$ )

RNA was extracted from cells using RNeasy kit (Qiagen, Germany), and reverse transcribed using SuperScript ${ }^{\circledR}$ III Reverse Transcriptase (Invitrogen, USA), according to the manufacturer's instructions. Quantitative real time reverse transcriptase polymerase chain reaction (qRT-PCR) was used to measure expression of markers for mammary stem cells

\section{RESULT}

Monkey breast-derived cells (MfMC) were cultured in selective medium specific for mammary epithelial cells enrichment. The MfMC cell population grown showed predominant epithelial-like morphology alongside fibroblast-like and adipocyte-like. The MfMC culture was successfully subcultured up to 3 passages with cell viability of $74 \%-88 \%$. Single cells of breast tissue has been successful culturing as monolayer and expressed mammary stem cells marker. These results indicate that our cell culture model is a reliable model for acquiring a population of cells with mammary stem cell properties and that these cultures may also serve as a reservoir (integrin beta-1; ITGB1 or CD29 and integrin alpha-6; ITGA6 or CD49f) and differentiation (Casein-beta; CSN2, signal transducer activator of transcription 5; STAT5 and GATA3). Reactions were performed using SsoFast ${ }^{\mathrm{TM}}$ EvaGreen ${ }^{\circledR}$ Supermix (Biorad, Hercules, Un) on the Icycler iQ5 (Biorad, USA). Relative expression was determined using the delta $\mathrm{Ct}$ method.

Data analysis was performed using JMP12 (SAS, Cary, NC). Log-transformation was done where appropriate to improve data normality. Data were back-transformed to original scale and presented as means \pm SEM. We performed ANOVA followed by multiple pairwise comparisons with Tukey Honestly Significant Difference (HSD) Test. For nonparametric data, analysis was performed by Kruskal-Wallis Test followed by Wilcoxon's Test for each pair.

from which more purified populations of stem cell populations can be isolated in the future.

Mammosphere derived from frozen cells MfMG successfully cultured to form sphere and multicellular observed starting on day 3, mammosphere number increased and showed increasing stem cell mammary gland marker such as ITGB1 and ITGA6. The 3D differentiation culture conditions produced organoid budding, categorized as coronal budding, sub-coronal budding, star-like budding, along with the formation of elongated ductal, and alveolar-like structures. Differentiation markers CSN2, GATA 3 and STAT5 were detected in both $2 \mathrm{D}$ and $3 \mathrm{D}$ models, indicating that differentiated cells were 
present. The expression of these markers were higher in the 3D model $(P<0.05)$.

\section{DISCUSSION}

Mammary gland stem cells is important for normal mammary morphogenesis as well as tumor initiation. In mouse model, the currently known cell surface markers to isolate MaSCs include CD24, ITGB1 and ITGA6 among others (Chen et al., 2017; Visvader, 2009), The use of these markers has been a beneficial tool to isolate and enrich MaSCs. Moreover, cells positive for ITGBI and ITGA6 were able to regenerate a functional mammary gland in murine model, which strongly supports the notion that these cells are part of a subpopulation that consists of mammary gland stem cells (Stingl et al., 2006; Shackleton et al., 2006). In humans, ITGA6 is among the known makers for MaSC with regenerative capacity, although the expression was also found in the epithelial luminal progenitor cells (Visvader, 2009). Here, we found the MfMC cultures were also positive for ITGBI and ITGA6, suggesting that a population of mammary stem cells likely exists in this primary cell culture model.

The cell culture were derived from breast tissue of nulliparous monkeys, as nulliparity is consistent with less differentiation of the mammary gland in monkeys and humans (Cline and Wood, 2008). Previously reported a study in monkeys that showed terminal end bud (TEB) number was greater with less differentiation of the breast (Dewi et al., 2013). TEB has been thought to be the site where most mammary stem cells exist, as this TEB will give rise to epithelial cells (Visvader, 2009). It is likely that choosing the right developmental stage of the breast as tissue source is key in gaining a culture model with ample mammary gland stem cell population to be enriched further. While our study indicates the likelihood of mammary stem cell population to be present in the cell culture derived from nulliparous breast tissue, pubertal breast may also serve as a good source for mammary stem cell culture since TEBs are most abundant in pre-menarchal breast (Dewi et al., 2013). Importantly, a subset of stem cell markers including ITGBI were expressed at the highest level during puberty compared to other life stages in monkeys (Stute et al., 2012). The use of monkey model may serve as a highly translational source to derive a culture model enriched for stem cells, especially from breast developmental stages that are ethically impossible to attain in humans.

Adult mammary stem cells hold an important role in the development of the mammary gland, and are also proposed to be cell-of-origin for some breast cancers (Wu et al., 2016). In that changes in numbers and/or physiology of the stem cell populations in mammary glands may be critical to the understanding of breast carcinogenesis there is a need for a reliable method to obtain adult mammary stem cell populations for use in invitro and ex-vivo studies. Here, a 3D mammosphere culture was formed using frozen primary mammary cells from the highly translational Mf model. Importantly, the cells 
used for these experiments were derived from small breast biopsy tissue samples, cultured as primary cells and cryopreserved into cell stocks. This refined method is significant as it allows for a minimally invasive and reliable approach to obtaining mammary stem cells that can be utilized over long periods of time.

The cells produced through these mammosphere cultures showed increase in expression levels of mammary stem cell markers, indicative of enhancement of adult mammary stem cell population, and were likewise able to differentiate and form organoids. Organoids can be expanded from embryonic cells, induced pluripotential stem (iPS) cells or from primary stem cells that have been isolated from organs. Notably, however, in vivo organs do not expand from single isolated stem cells, and therefore the mechanisms that drive the formation of stem cell organoids may be distinct from organogenesis in vivo (Shamir and Ewald, 2014).

The organoid morphology can be used to define the necessary and sufficient components model.

\section{REFERENCES}

Chen X, Liu Q, Song E. 2017. Mammary stem cells : angels or demons in mammary gland? Signal Transduction and Targeted Therapy 2:16038.

Cline JM, Wood CE. 2008. The Mammary Glands of Macaques. Toxic. path.36:134s-141s.

Dewi FN, Wood CE, Lees CJ, Willson CJ, Register TC, Tooze JA, Franke AA, to replicate the structure and function of mammary gland. Culture of Mf mammospheres in matrigel resulted in organoid budding in this study. Morphologically, these organoids contained diverse epithelial cell types that were organized into their normal spatial configurations as observed in vivo. Our results show that mammospheres can be reliably obtained from cryopreserved Mf mammary gland cell cultures initially derived from small mammary gland biopsies; mammosphere culture enriches the previously-cryopreserved Mf mammary gland cell cultures for mammary stem cell populations; and the Mf mammary stem cells have the capability to further differentiate down multiple lineages when grown in mammary epithelial cell growth medium or matrigel. In short, the Mf mammosphere model is likely to be highly useful for a variety of future studies involving stem cell differentiation and/or breast carcinogenesis. Further studies are currently planned to better characterize and advance this cell culture

Cline JM. 2013. Dietary soy effects on mammary gland development during the pubertal transition in nonhuman primates. Cancer Prev. Res. 6:832-842.

Dey D, Saxena M, Paranjape AN, Krishnan V, Giraddi R, Kumar MV, Mukherjee G, Rangarajan A. 2009. Phenotypic and Functional Characterization of 
Human Mammary Stem/Progenitor Cells in Long Term Culture. PLoS ONE 2009;4.

LaMarca HL, Rosen JM. 2008. Minireview: Hormones and mammary cell fate What will I become when I grow up? Endocrinology 149:4317-4321.

Liu S, Dontu G, Wicha MS. 2005. Mammary stem cells, self-renewal pathways, and carcinogenesis. Breast Cancer Res.7:86-95.

Lombardo Y, Giorgio A de, Coombes CR, Stebbing J, Castellano L. 2015. Mammosphere Formation Assay from Human Breast Cancer Tissues and Cell Lines. J. Vis.Exp. 2015;1-5. Shackleton M, Vaillant F, Simpson KJ, Stingl J, Smyth GK, Asselin-Labat ML, Wu L, Lindeman GJ, Visvader JE. 2006. Generation of a functional mammary gland from a single stem cell. Nature 439, 84-88.

Shamir ER, Ewald AJ. Three-dimensional organotypic culture: Experimental models of mammalian biology and disease Three - dimensional organotypic culture: experimental models of mammalian biology and disease. Nat. Publ. Gr. 15: 647-664.

Stingl J, Eirew P, Ricketson I, Shackleton M, Vaillant F, Choi D, Li HI, Eaves CJ. 2006. Purification and unique properties of mammary epithelial stem cells. Nature 439:993-997.

Stute P, Sielker S, Wood CE, Register TC, Lees CJ, Dewi FN, Williams JK, Wagner JD, Stefenelli U, Cline JM. 2012. Life stage differences in mammary gland gene expression profile in non-human primates. Breast Cancer Res.Treat. 133;617-634.

Visvader JE. 2009. Keeping abreast of the mammary epithelial hierarchy and breast tumorigenesis. Genes Dev. 23, 25632577.

Wu A, Dong Q, Gao H, Shi Y, Chen Y, Zhang F, Bandyopadhyay A, Wang D, Gorena KM, Huang C, et al. Characterization of mammary epithelial stem/progenitor cells and their changes with aging in common marmosets. Scientific reports. Nature Publishing Group, 6(August), p. 32190 